\title{
Additional evaluation of the point-of-contact circulating cathodic antigen assay for Schistosoma mansoni infection
}

\section{Pauline N. M. Mwinzi ${ }^{1}$, Nupur Kittur ${ }^{2}$, Elizabeth Ochola ${ }^{1}$, Philip J. Cooper ${ }^{3,4}$, Carl H. Campbell Jr. ${ }^{2}$, Charles H. King ${ }^{5}$ and Daniel G. Colley ${ }^{2,6 *}$}

\author{
1 Neglected Tropical Diseases Research Unit, Center for Global Health Research, Kenya Medical Research Institute, Kisumu, Kenya \\ ${ }^{2}$ Center for Tropical and Emerging Global Diseases, University of Georgia, Athens, GA, USA \\ 3 Institute of Infection and Immunity, St George's University of London, London, UK \\ ${ }^{4}$ Centro de Investigación en Enfermedades Infecciosas y Crónicas, Pontificia Universidad Católica del Ecuador, Quito, Ecuador \\ ${ }^{5}$ Center for Global Health and Diseases, Case Western Reserve University School of Medicine, Cleveland, OH, USA \\ ${ }^{6}$ Department of Microbiology, University of Georgia, Athens, GA, USA
}

\section{Edited by:}

Rafaella Fortini Grenfell E. Queiroz, Fundação Oswaldo Cruz, Brazil

Reviewed by:

Diana Cordeiro Taboada, Fundação

Oswaldo Cruz, Brazil

Cornelis Hendrik Hokke, Leiden

University Medical Center,

Netherlands

*Correspondence:

Daniel G. Colley, Center for Tropical and Emerging Global Diseases,

University of Georgia, 500 DW

Brooks Drive, Room 303B Coverdell

Center, Athens, GA 30602, USA

e-mail:dcolley@uga.edu
Studies of the urine-based point-of-contact cathodic circulating antigen test (POC-CCA) in Schistosoma mansoni-endemic settings in Africa indicate it has good sensitivity in detecting infections, but in areas of low prevalence, the POC-CCA can be positive for persons who are egg-negative by Kato-Katz stool assays. We examined the POC-CCA assay for: (a) batch-to-batch stability; (b) intra-reader and inter-reader variability; (c) day-to-day variability compared to Kato-Katz stool assays, and (d) to see if praziquantel (PZQ) treatment converted Kato-Katz-negative/POC-CCA positive individuals to POC-CCA negativity. We found essentially no batch-to-batch variation, negligible intra-reader variability (2\%), and substantial agreement for inter-reader reliability. Some day-to-day variation was observed over 5 days of urine collection, but less than the variation in Kato-Katz stool assays over 3 days. To evaluate the effect of treatment on Kato-Katz(-)/POC-CCA $(+)$ children, 149 children in an area of $10-15 \%$ prevalence who were Kato-Katz(-) based on 3 stool samples but POC$\mathrm{CCA}(+)$ were enrolled. Seven days after treatment (PZQ $40 \mathrm{mg} / \mathrm{kg}$ ) samples were again collected and tested. Almost half (47\%) POC-CCA positive children turned negative. Those still POC-CCA positive received a second treatment, and 34\% of them turned POC-CCA negative upon this second treatment. Most who remained POC-CCA positive shifted each time to a "lesser" POC-CCA "level of positivity." The data suggest that most Kato-Katznegative/POC-CCA positive individuals harbor low-intensity infections, and each treatment kills all or some of their adult worms. The data also suggest that when evaluated by a more sensitive assay, the effective cure rates for $\mathrm{PZQ}$ are significantly less than those inferred from fecal testing. These findings have public health significance for the mapping and monitoring of Schistosoma infections and in planning the transition from schistosomiasis morbidity control to elimination of transmission.

Keywords: schistosomiasis, diagnosis, POC-CCA, praziquantel, Kato-Katz, Schistosoma mansoni

\section{INTRODUCTION}

WHO guidelines for control and elimination of schistosomiasis involve pre-treatment and periodic post-treatment evaluations of the prevalence of Schistosoma infections to inform programmatic decisions on whom, and how often to treat within endemic areas (1). For Schistosoma mansoni, this has usually involved mapping by examinations for parasite eggs in the stool, most often by the KatoKatz thick-smear microscopic technique (2). By making stool exams more feasible on a population level, the Kato-Katz technique revolutionized the process of obtaining intensity and prevalence data from large numbers of people. This assay worked very well for control programs in areas of high intensity and corresponding high prevalence. It was used extensively and became established as part of WHO guidelines for morbidity control programs, with prevalence estimates usually based on examination of one stool per subject, using two separate Kato-Katz slides. In situations with high-mean intensities of infection (and of corresponding high prevalence), the Kato-Katz is believed to provide close to true prevalence estimates of local infection for schistosomiasis control programs (3). Research programs also use it extensively - most often testing three consecutive stools, two Kato-Katz slides on each stool for greater accuracy of diagnosis. However, the Kato-Katz assay has documented day-to-day and intra-stool variability, especially in communities with moderate-to-low intensity of infection and corresponding low prevalence. Day-to-day variation is biological (4), and intra-stool variation is related to sampling error based on the amount of stool evaluated (5). Thus, the Kato-Katz test is acknowledged to be relatively insensitive at lower levels of intensity of infection, often corresponding to lower prevalence as well. Over the course of a control program, the ability of the KatoKatz assay to provide true prevalence estimates will become less and less as the population mean intensity of infection decreases 
(6-14). Over 20 years ago, de Vlas et al. $(3,15,16)$ published a series of statistical reviews that showed that programmatic dependence on a single stool examination led to underestimates of true prevalence and that observations based on such surveys could result in inaccurate allocation of control measures.

Currently, an ideal diagnostic tool for implementing schistosomiasis control programs would be one that could be easily used for regional mapping to initiate a "gaining control" program. In this scenario, low sensitivity may be acceptable but high specificity is good. A tool for monitoring the post-treatment impact of an initial "gaining" or ongoing "sustaining" control program would need to have high sensitivity for low intensity infections and retain high specificity. It would serve as the tool that determines if it is time to switch MDA protocols or add adjunct strategies, such as snail control, behavioral change, or implementation of water and sanitation measures, as prevalence and intensities decrease. The tool used to determine when it is time to switch from a standard control program to a more aggressive elimination program must have very high sensitivity, and ideally high specificity too. A postelimination surveillance tool would require excellent sensitivity and excellent specificity based on exposure or infection and allow high throughput.

Over the last several years, multiple studies have evaluated a commercially available, urine-based, point-of-contact (POC) cassette assay that detects circulating cathodic antigen (CCA). CCA is derived from the gut of adult schistosomes that are established in the human circulation $(8,17-22)$. The antigen is eliminated by the kidneys into the urine where it can be detected by a standardized immunoassay. In general, the results of earlier evaluations indicate that the POC-CCA assay is suitable for the prevalence mapping required for regional schistosomiasis morbidity control programs (21). For this purpose, POC-CCA testing appears just as good as or better than using Kato/Katz assays. With the additional advantage of being urine-based, it is able to be done on-site, and appears to be more sensitive for detection of S. mansoni infection at low egg intensities $(8,21,22)$. The POC-CCA is now being used in several countries, but its performance should be continually reassessed to determine its potential flaws and limitations as control programs mature. Because there is no sensitive and specific "gold standard" for diagnosis of active S. mansoni infection, there remain questions about the overall specificity and sensitivity of the POC-CCA assay and thus about its use in place of the Kato-Katz assay for programmatic decision making. One of the questions revolves around observer readings of faint diagnostic bands, considered positive by the manufacturer and called "trace" results. However, it has also been suggested that information can be obtained based on the differential intensity of a positive POC-CCA band to indicate, in semi-quantitative fashion, levels of intensity of infection $(13,21,23)$. In addition, it is questioned if the POC-CCA assay could prove more reliable than the Kato-Katz as an indicator of the effectiveness of drug treatment of S. mansoni infections (23). In the present report, we address some of these questions based on prospective laboratory and field trials in an S. mansoni-endemic area of Kenya, on additional testing data from a non-endemic site in coastal Ecuador, and previously reported data (21) from a non-endemic area of Ethiopia.

\section{MATERIALS AND METHODS ETHICS STATEMENT AND PARTICIPANT SELECTION}

The study protocol was approved by the scientific steering committee (SSC) of the Kenya Medical Research Institute (KEMRI, SSC number 1820), the Ethical Review Committee (ERC) of KEMRI, the Institutional Review Boards (IRB) of the Centers for Disease Control and Prevention (CDC), the Pontificia Universidad Catolica del Ecuador, Quito, Ecuador, the Aklilu Lemma Institute of Pathobiology, Addis Ababa University of Ethiopia and of the University of Georgia. Parents/guardians, and participating children were informed about the purpose and procedures of the study. Written informed consent was obtained from the parents/guardians and the children participating in the study gave assent prior to study enrollment. All samples obtained in the study were coded and treated confidentially.

\section{STUDY POPULATION AND SAMPLE COLLECTION}

Most of this study was conducted in schools near the shores of Lake Victoria, western Kenya from January 2013 to April 2014. Studies in areas known not to be endemic for schistosomiasis but endemic for soil-transmitted helminths (STHs) were done in Ethiopia [as previously reported; (21)], and in the coastal region of Ecuador. From each school, children above the age of 6 years for whom consent and assent had been obtained were selected for study inclusion. All children who were positive for schistosome infection were treated with praziquantel (PZQ) $(40 \mathrm{mg} / \mathrm{kg})$ and children positive for STHs were given $400 \mathrm{mg}$ Albendazole as a single dose. All contact with the children was done at the school.

\section{LABORATORY METHODS AND STUDY DESIGNS Kato-Katz testing}

Stool samples collected were processed within $24 \mathrm{~h}$ of collection using the Kato-Katz thick-smear technique in duplicate (normally three stools, six slides) for the quantitative determination of $S$. mansoni and STHs (2). Each slide was read within $1 \mathrm{~h}$ of preparation to diagnose presence of hookworm, Ascaris lumbricoides, and Trichuris trichiura by two well-trained microscopists. The $S$. mansoni readings were performed at least $24 \mathrm{~h}$ after slide preparation and recorded as number of eggs per gram of stool (EPG) (2). The results of all slides read were averaged and the intensity of infection classified according to the WHO guidelines (24). STH infections were simply categorized as being either positive or negative.

\section{POC-CCA testing}

Urine samples were collected and tested in the laboratory for positivity and band intensity by the commercially available POC-CCA assay, according to manufacturer's instructions (Rapid Diagnostics, Pretoria). Briefly, one drop of urine was added to the well of the testing cassette and allowed to absorb. Once it was absorbed, a drop of buffer (included in the kit) was added into the same well and the assay was allowed to develop for $20 \mathrm{~min}$, at which time the results were read. The tests were considered invalid if an internal control band did not appear or if the tests were left to develop for more than $25 \mathrm{~min}$ after addition of the buffer before being read. In these few cases, the samples were re-run with a new test cassette and scored appropriately. To score the intensity of the POC-CCA assay results, the intensity of the test band was compared to that 
of the control band. Positive results were given a score of "trace" if the band was barely visible, $1+$ if the test band was less intense than the control band, $2+$ if the test band was of equal intensity as the control band, and $3+$ if the test band was more intense than the control band.

\section{Cassette batch variation}

Five separate batches of CCA test kits were used for this study. Three readers were provided with 10 urine samples from subjects who either did not have S. mansoni infections or had infections that ranged from low to moderate intensity based on Kato-Katz stool examinations. Each of the 3 readers read the 10 urine samples using 10 POC-CCA cassettes from Box \# 1. The urine samples were shuffled and then read again using 10 cassettes from Box \# 2; this retesting of the 10 test urines continued using cassettes from the remaining boxes, 10 per box, to Box \# 5 .

\section{Intra-reader reliability}

Five readers were provided with 10 urines that ranged from negative to moderately positive, as described above. In Round 1 , each reader read urine results using 10 individual POC-CCA cassettes read at $20 \mathrm{~min}$. For Round 2, the cassettes were randomly rearranged, and each reader re-read and re-scored the same (masked) cassettes for a second time within $\sim 5 \mathrm{~min}$, to keep within the time limit for valid cassette readings.

\section{Inter-observer reliability}

Five readers were provided with 30 urines that ranged from negative to moderately positive, as described above. The five readers read the urines on POC-CCA cassettes independently and the results were recorded without discussion.

\section{Day-to-day variability}

Seventy-three participants were recruited for this study. Five consecutive urine samples for POC-CCA and three consecutive stool samples for Kato-Katz were collected and assessed to determine if there were any fluctuations in the POC-CCA or Kato-Katz readings based on the different days of collection.

\section{Post-treatment readings and re-treatments}

One hundred forty-nine children from schools that had received two or three rounds of annual MDA with PZQ and who were POC-CCA positive but Kato-Katz negative (based on a single urine sample and three stool samples) were enrolled at baseline, and treated with PZQ based on the POC-CCA positive results. Seven days later, a single urine and stool sample were collected and tested by POC-CCA and Kato-Katz, respectively. Children who were still POC-CCA positive were treated for a second time, and 7 days later urine and stool samples were again collected and assayed. Children who were still POC-CCA positive were again treated for a third time without further testing. A schematic diagram of the post-treatment study is shown in Figure 1.

\section{DATA ANALYSES}

Data collected from evaluations of school children included egg intensity readings and POC-CCA cassette band readings and were entered into an Excel database and analyzed using SAS statistical software (SAS, Cary, NC, USA). Fleiss kappa statistical analysis (25)

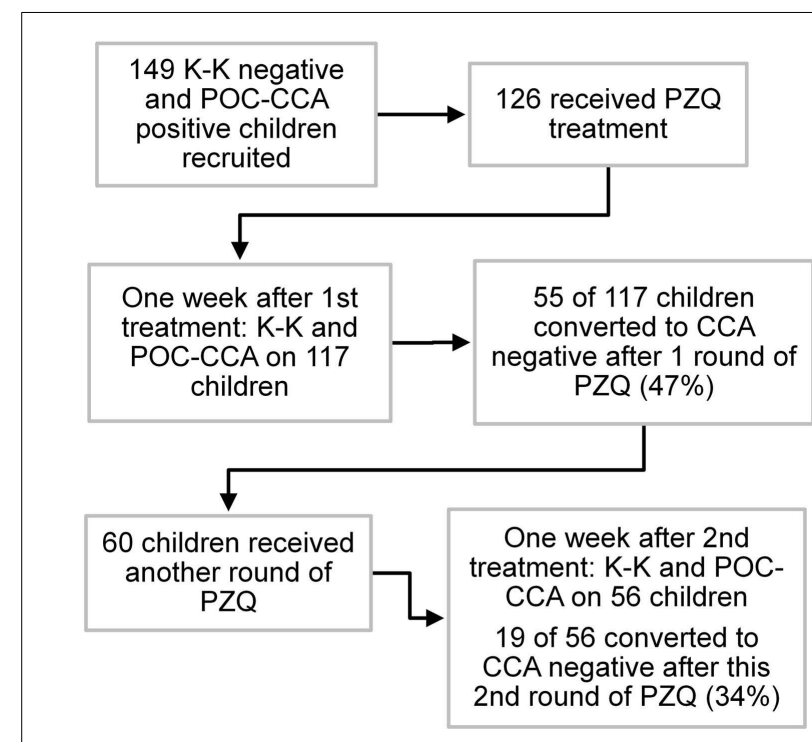

FIGURE 1 | Flow diagram of post-praziquantel treatment study, Kenya

was used to measure the level of inter-reader agreement among five readers, with values of $0.00-0.20$ considered slight, $0.21-0.40$ fair, 0.41-0.60 moderate, $0.61-0.80$ substantial, and $0.81-1.00$ almost perfect agreement (26). The estimated diagnostic accuracy of the Kato-Katz and POC-CCA tests was compared using Bayesian latent class modeling software (27), or by measuring detection accuracy against a "combined gold standard." The combined gold standard was defined as any one positive test out of all eight tests (five consecutive CCA and three Kato/Katz tests) for any individual subject. In a separate analysis, Spearman's rank correlation was used to compare trace, $1+, 2+, 3+$ band intensity on the POC-CCA assay with estimated infection intensity determined by Kato-Katz egg counts. Fleiss kappa and Spearman's rho were analyzed using IBM SPSS 21.

\section{RESULTS}

\section{CASSETTE BATCH VARIATION}

When different batches of the cassettes provided by the manufacturer were tested on the same 10 urine samples, there was no real variation between them. Nine of 10 samples showed the same results across all 5 batches for all 3 readers. Only one sample gave varying results when cassettes were taken from different batches, being negative for some lots and trace (positive) on others. This same variation occurred for all three readers.

\section{INTRA-READER RELIABILITY}

When 5 trained readers read two rounds of the same 10 specimens, there was only 1 reading of the 50 pairs of repeat readings that was not replicated. That one was read as negative the first time and as trace (positive) when it was re-read by one of the readers. This corresponds to a $2 \%$ intra-reader variation.

\section{INTER-READER RELIABILITY}

When 5 trained readers were evaluated in regard to the reliability of their readings of the same 30 specimens and the results 
analyzed by Fleiss kappa statistics the overall Fleiss kappa value for inter-reader agreement of the POC-CCA scores was 0.70 (95\% confidence interval: $0.58,0.81 ; P<001)$. This indicates substantial agreement between the five different readers.

\section{DAY-TO-DAY VARIABILITY}

Seventy-three participants each had POC-CCA tests on five consecutive days' urines; and Kato-Katz tests on three consecutive days' stools (two slides each) to evaluate $S$. mansoni prevalence. This study sample was representative of a moderate-to-high prevalence area ( $44 \%$ on the first Kato-Katz stool/two slides). As per the manufacturer's instructions, "Trace" readings were considered positive. For each assay, prevalence estimates based on single tests did not vary significantly between days, ranging between 81 and $88 \%$ for the five consecutive POC-CCA and between 41 and $44 \%$ for three consecutive Kato/Katz tests. Almost all (94\%) of children were positive for at least one of the five POC-CCA tests while $70 \%$ were positive for the at least one of Kato/Katz tests (Table 1).

When the first three CCA consecutive tests were taken together, $82 \%$ of participants had consistent daily results compared to only $49 \%$ of participants with consistent daily results by Kato-Katz assays. Mixed results (i.e., both positive and negative results for the same participant) were observed in $18 \%$ of the participants for the POC-CCA tests but in $48 \%$ of Kato-Katz tests. Thus, while there was some day-to-day variation for both assays, the Kato-Katz varied much more in terms of diagnosing the presence of infection than the POC-CCA (Table 2).

The sensitivity and specificity of the POC-CCA assay were evaluated against an artificial "gold standard" of any one positive test of the combined eight tests (five POC-CCA and three Kato-Katz). These analyses indicated that the POC-CCA assay provided high levels of both sensitivity and specificity, as shown in Table 3.

When the POC-CCA assay was tested in areas in Ethiopia and Ecuador that had never been endemic for schistosomiasis but were endemic for STH, only one presumably false positive test was found among 100 participants in Ethiopia (21), while none in the 143 participants from Ecuador was positive.

A Bayesian latent class model (BLCM) was developed using data from all 73 subjects in this part of the study, assessing the

Table 1 | Schistosomiasis prevalence based on one or several POC-CCA and Kato-Katz tests among 73 school children, Kenya.

Prevalence, $N(\%)$

\section{POC-CCA}

Day

1

2

3

4

5

At least 1 of 5 CCA tests positive

Kato-Katz

Day

$\begin{array}{ll}1 & 32(44) \\ 2 & 31(43) \\ 3 & 30(41) \\ -K \text { tests positive } & 51(70)\end{array}$

$59(81)$

$62(85)$

$62(85)$

$62(85)$

$64(88)$

69 (94)

$2(44)$

$30(41)$

At least 1 of $3 \mathrm{~K}-\mathrm{K}$ tests positive most likely performance characteristics of 1 up to 5 daily POCCCA results, and 1 up to 3 daily Kato-Katz stool results, guided by the POC-CCA specificity data Ethiopian and Ecuadorian children described above (Figure 2). By this analysis, it was estimated that the sensitivity of a single POC-CCA assay was likely to be much greater than that of a single Kato-Katz test, and even more sensitive than performing three Kato-Katz tests in detecting S. mansoni infection in the study population. The estimated specificity for both POC-CCA and Kato-Katz assays approached 100\%.

Infection intensity using POC-CCA can be determined only semi-quantitatively by comparing the density of the test line that develops to the control line provided in the cassette. Yet, we found a good correlation between egg intensity determined by Kato-Katz as compared to POC-CCA scores based on readings the intensities of the positive bands. All the 10 participants who had moderate or high egg counts by Kato-Katz had an unambiguously positive PCO-CCA result. There was a significant correlation between a participant's mean EPG and their CCA score on all of the tested days (Figure 3).

\section{POST-TREATMENT READINGS AND RE-TREATMENTS}

For this phase of the study, 149 children who had tested negative by Kato-Katz (3 stools/2 slides each) but positive by POC-CCA (tested on a single urine) were selected from an area that had achieved 10-15\% overall S. mansoni prevalence (by Kato-Katz) following 2 or 3 rounds of MDA with PZQ. One week after testing, 126 of these children were treated with PZQ. Of those not lost to follow-up, 55 of 117 (47\%) became POC-CCA negative on repeat testing performed 1 week post-treatment. Those who remained POC-CCA positive (and were available) were treated a second time, and 7 days later, 19 of 56 (34\%) children were negative

Table 2 | Consistency of results for three consecutive diagnostic tests for schistosomiasis on $\mathbf{7 3}$ school children, Kenya.

$N(\%)$

Result of 3 POC-CCA tests

All 3 negative

All 3 positive (Trace/1/2/3)

$55(75)$

Mixed positive and negative results

$13(18)$

Result of 3 Kato-Katz tests (2 slides taken together)

All 3 negative

$21(29)$

All 3 positive

$15(20)$

Mixed positive and negative results

$35(48)$

Table 3 | Sensitivity and specificity analysis of POC-CCA one day testing evaluated against a combined gold standard of at least 1 of 8 tests (5 POC-CCA and 3 Kato-Katz) being positive.

\begin{tabular}{lcc}
\hline & Sensitivity, $\% \mathbf{~ ( 9 5 \% ~ C l )}$ & Specificity, $\% \mathbf{~ ( 9 5 \% ~ C l )}$ \\
\hline CCA day 1 & $85.5(74.9-2.8)$ & $100(40.2-100)$ \\
CCA day 2 & $89.9(80.2-95.8)$ & $100(40.2-100)$ \\
CCA day 3 & $89.9(80.2-95.8)$ & $100(40.2-100)$ \\
CCA day 4 & $89.9(80.2-95.8)$ & $100(40.2-100)$ \\
CCA day 5 & $92.7(83.9-97.6)$ & $100(40.2-100)$
\end{tabular}




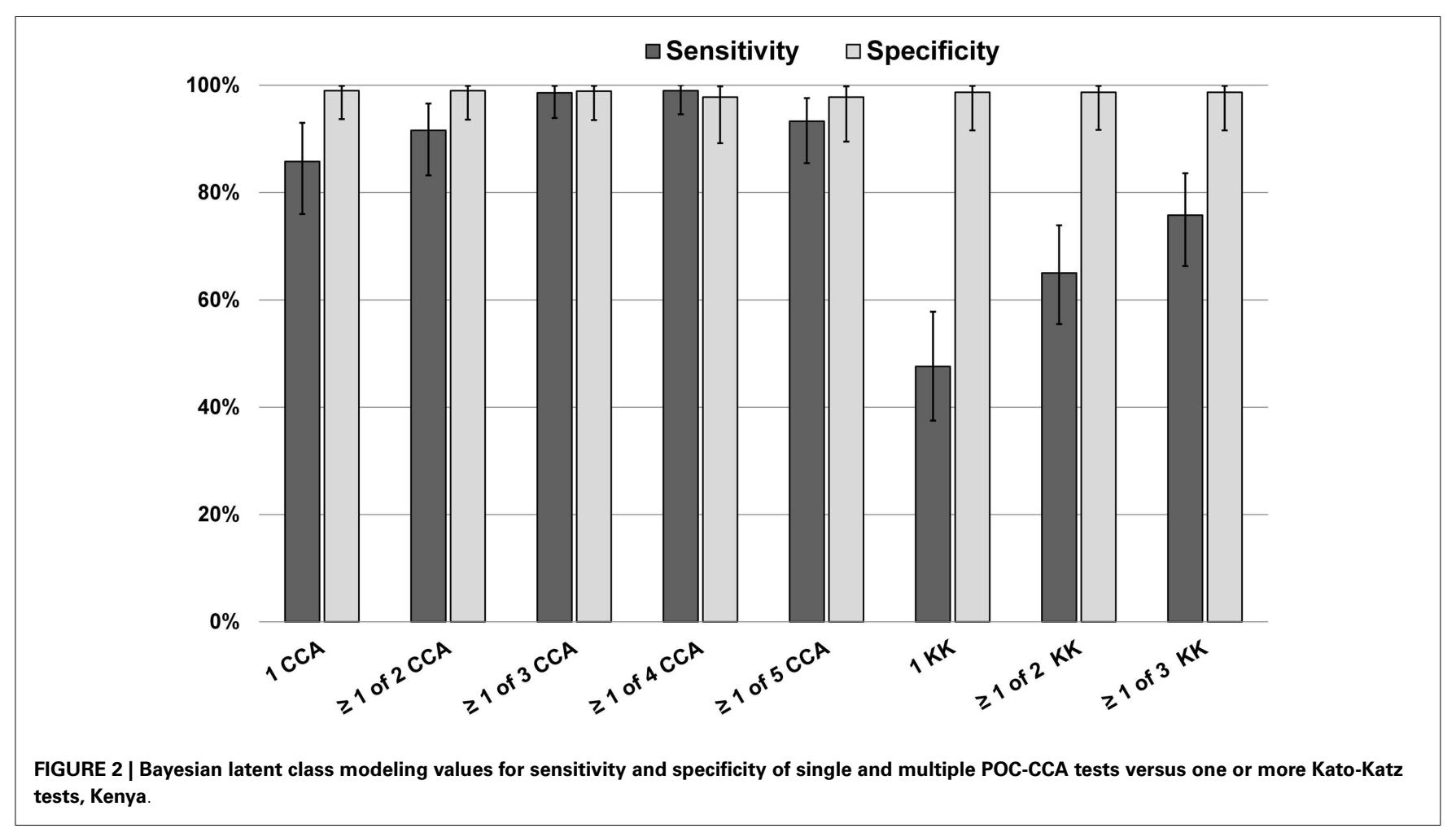

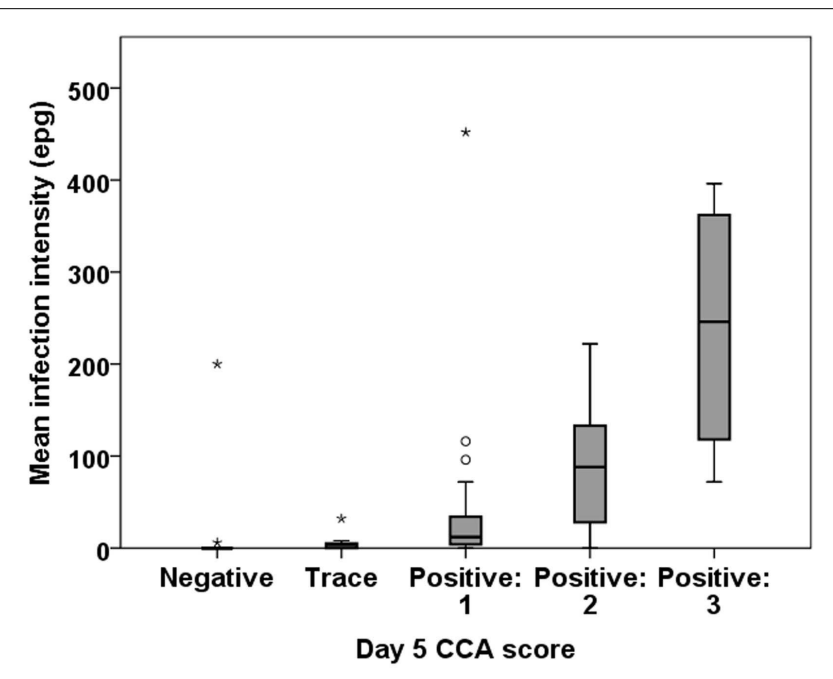

FIGURE 3 | Correlation between intensity of infection by Kato-Katz (EPG) and band intensity by POC-CCA. Representative boxplot is shown from day 5 POC-CCA test. There is a significant association between the EPG and CCA band intensity: Spearman's rho $=0.601(P<0.001)$.

based on follow-up POC-CCA testing at that time. The Kato-Katz assays of these children, who were selected as Kato-Katz negative, remained negative after each treatment round.

\section{DISCUSSION}

Many schistosomiasis control programs in Sub-Saharan Africa are now at the point of mapping and moving toward gaining control by mass drug administration. Some are sustaining control at a moderate level but will eventually wish to move toward elimination of transmission of S. mansoni infection. The sensitivity of the assay used to evaluate prevalence in these different phases becomes critical, as the current Kato-Katz test is known to become significantly less sensitive as egg count intensities decrease (23, 24, 28-33). Our data and those from other studies indicate that the POC-CCA assay is more sensitive than the Kato-Katz assay $(13,22)$. Therefore, in moderate to lower prevalence and intensity areas, POC-CCA will indicate higher prevalence levels than the Kato-Katz. Since prevalence estimates impact programmatic guidelines for coverage, questions have been raised about the true sensitivity and specificity of POC-CCA readings. For this reason, we undertook further evaluation of this urine-based, commercially available assay.

Our study found little or no variability of performance between batches of the POC-CCA cassettes, and intra-reader variability was minimal when trained technicians performed the assay. In addition, Fleiss kappa statistics indicated substantial agreement in regard to inter-reader reliability among 5 trained readers reading 30 specimens (Fleiss kappa value $=0.70$ ). We had previously observed that when non-technician field workers were minimally trained and then asked to read multiple POC-CCA tests, there was considerable variation in the readings. Since there was little or no variability seen when trained readers (laboratory technicians) were used, this highlights the need for adequate training prior to use of the POC-CCA assay.

Day-to-day variability in per-subject prevalence, based on readings from 3 successive days of Kato-Katz or 5 days of POC-CCA, was minimal and almost all variation occurred at the very low 
intensity end of the CCA test spectrum, involving "trace" readings. Also, based on the first 3 days of testing run in parallel, the day-to-day variability of the POC-CCA readings (18\%) was much less than those of the Kato-Katz (48\%).

Our post-treatment data suggest that, based on the estimated sensitivity of the POC-CCA assay, many of the Kato-Katznegative/POC-CCA positive results reflect continued infection, i.e., these subjects have remaining worms that continue to make detectable levels of CCA. This means that currently accepted PZQ cure rates for Schistosoma infection are likely to be significantly overestimated, based on the post-treatment inaccuracy of a lowersensitivity assay, the Kato-Katz. PZQ is typically said to produce 70-90\% cure rates as evaluated by Kato-Katz assays (34-37). If, in fact, many treated individuals continue to harbor low-intensity infection (as determined by a more sensitive assay such as the POC-CCA) this suggests the need to treat many more people, more often, for persistent infection. Such a shift in recommendations would constitute a significant alteration in programmatic decision-making algorithms from when Kato-Katz testing is used as the sole diagnostic criterion.

Our data and those of others (13) indicate that the POC-CCA assay can be used in post-treatment studies and programs, and is indeed more suitable for post-treatment evaluations where the goal is to detect uncured persons. In an earlier drug efficacy study, the POC-CCA was found to be more sensitive than six Kato-Katz slides (two/slides on three stools) at 4-weeks-post-praziquantel, and again at 6 months post-praziquantel (13). We have shown that, following treatment, there are still those with positive POCCCA readings who are K-K negative - and these drop by roughly half upon a second treatment and again after another retreatment. Those who do not convert to POC-CCA negative upon these treatments decreased the intensity of their CCA bands, perhaps indicating successive partial cures (Figure 4).

Locations that were endemic for STH but not human schistosomes (areas of Ethiopian and Ecuador) yielded only one "trace" POC-CCA positive (from Ethiopia) child out of 243 children tested, confirming the high specificity of this assay.

In conclusion, our data show that most (if not all) Kato-Katznegative/POC-CCA positive individuals in endemic areas are likely to harbor low level infections with $S$. mansoni. While not a perfect test (as seen in the day-to-day variability) the POC-CCA assay appears to be highly suitable for initial mapping of S. mansoni areas, and for subsequent post-treatment programmatic decision making. The fact that it indicates higher prevalences than the less sensitive Kato-Katz in areas with low-average intensity should now be factored into how treatment decisions will be made. Our data bring up the possibility that many people in endemic areas are harboring low levels of worms that are producing few, if any, eggs on a daily basis. This raises an issue that is seldom voiced, but may be quite important in programmatic thinking. What are the consequences of such a scenario? If few or no eggs are being released every day, does that level of infection still lead to morbidity? If few or no eggs are being released, it would seem that the implications for transmission would be important. We do not yet have answers to these questions, but believe they need to be discussed. They will continue to arise as the POC-CCA and other more sensitive assays are introduced and employed more widely in the field.

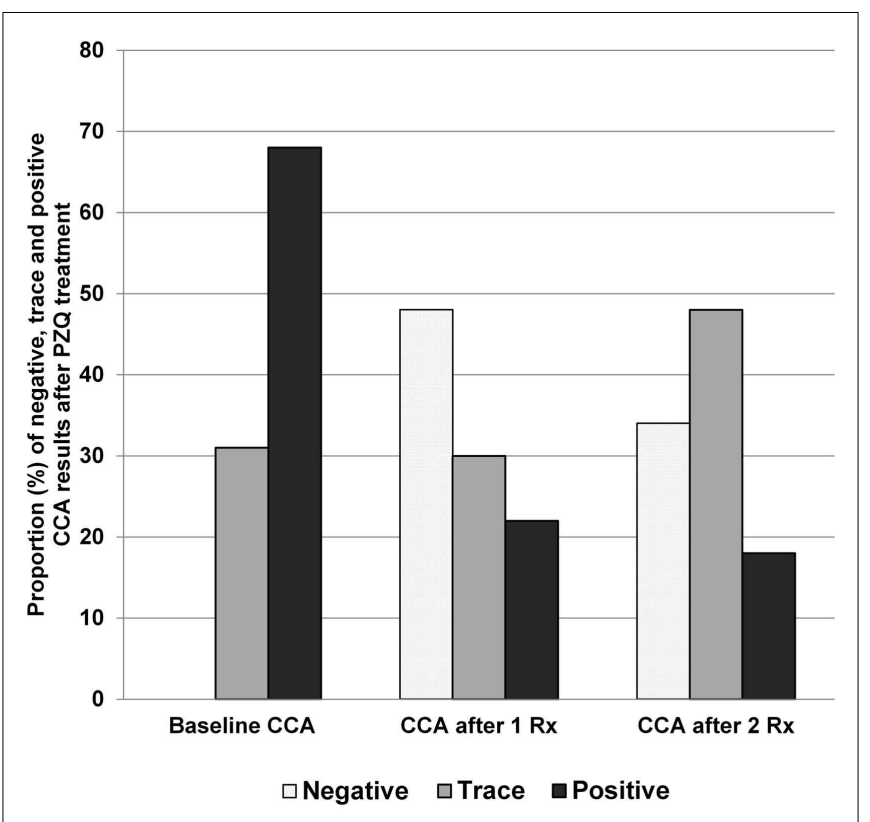

FIGURE 4 | POC-CCA results following two rounds of praziquantel treatment, Kenya.

\section{AUTHOR CONTRIBUTIONS}

The study was designed by DC, NK, CK, CC, and PM. EO and PC coordinated field activities and laboratory work. NK and CK analyzed the data. All authors actively contributed to the interpretation of the findings, data compilation, and manuscript preparation. All authors read and approved the final manuscript.

\section{ACKNOWLEDGMENTS}

We thank the parents/guardians, head teachers, teachers, and pupils in each of the schools that participated in this study. We also thank Dr. Berhanu Erko of Aklilu Lemma Institute of Pathobiology, Addis Ababa University for the data from the Ethiopian site non-endemic for schistosomiasis. This paper is published with the permission of the Director of the Kenya Medical Research Institute. This study received financial support from the University of Georgia Research Foundation, Inc., which was funded by the Bill and Melinda Gates Foundation for the SCORE project. The funders had no role in the study design, data collection and analysis, decisions to publish, or preparation of the manuscript.

\section{REFERENCES}

1. World Health Organization. Helminth Control in School-Age Children: A Guide for Managers of Control Programmes. Geneva: World Health Organization (2011). Available from: http://whqlibdoc.who.int/publications/2011/ 9789241548267_eng.pdf

2. Katz N, Chaves A, Pellegrino J. A simple device for quantitative stool thicksmear technique in Schistosoma mansoni. Rev Inst Med Trop Sao Paulo (1972) 14:397-400.

3. de Vlas SJ, Gryseels B, van Oortmarssen GJ, Polderman AM, Habbema JD. A pocket chart to estimate true Schistosoma mansoni prevalences. Parasitol Today (1993) 9(8):305-7.

4. Hubbard A, Liang S, Maszle D, Qiu D, Gu X, Spear RC. Estimating the distribution of worm burden and egg excretion of Schistosoma japonicum by 
risk group in Sichuan Province, China. Parasitology (2002) 125(Pt 3):221-31. doi:10.1017/S003118200200207X

5. Krauth SJ, Coulibaly JT, Knopp S, Traoré M, N'Goran EK, Utzinger J. An indepth analysis of a piece of shit: distribution of Schistosoma mansoni and hookworm eggs in human stool. PLoS Negl Trop Dis (2012) 6(12):e1969. doi:10.1371/journal.pntd.0001969

6. Nikolay B, Brooker SJ, Pullan RL. Sensitivity of diagnostic tests for human soil-transmitted helminth infections: a meta-analysis in the absence of a true gold standard. Int J Parasitol (2014) 44(11):765-74. doi:10.1016/j.ijpara.2014. 05.009

7. Zhu HQ, Xu J, Zhu R, Cao CL, Bao ZP, Yu Q, et al. Comparison of the miracidium hatching test and modified Kato-Katz method for detecting Schistosoma japonicum in low prevalence areas of China. Southeast Asian J Trop Med Public Health (2014) 45(1):20-5.

8. Adriko M, Standley CJ, Tinkitina B, Tukahebwa EM, Fenwick A, Fleming FM, et al. Evaluation of circulating cathodic antigen (CCA) urine-cassette assay as a survey tool for Schistosoma mansoni in different transmission settings within Bugiri District, Uganda. Acta Trop (2014) 136:50-7. doi:10.1016/j.actatropica. 2014.04.001

9. Qian MB, Yap P, Yang YC, Liang H, Jiang ZH, Li W, et al. Accuracy of the KatoKatz method and formalin-ether concentration technique for the diagnosis of Clonorchis sinensis, and implication for assessing drug efficacy. Parasit Vectors (2013) 6(1):314. doi:10.1186/1756-3305-6-314

10. Othman RA. Indirect haemagglutination test and ELISA as compared to Kato thick-smear in diagnosing Schistosoma mansoni. J Egypt Soc Parasitol (2013) 43(3):841-8. doi:10.12816/0006439

11. Espírito-Santo MC, Alvarado-Mora MV, Dias-Neto E, Botelho-Lima LS, Moreira JP, Amorim M, et al. Evaluation of real-time PCR assay to detect Schistosoma mansoni infections in a low endemic setting. BMC Infect Dis (2014) 14(1):558. doi:10.1186/s12879-014-0558-4

12. Sayasone S, Utzinger J, Akkhavong K, Odermatt P. Repeated stool sampling and use of multiple techniques enhance the sensitivity of helminth diagnosis: a crosssectional survey in Southern Lao people's democratic republic. Acta Trop (2015) 141:315-21. doi:10.1016/j.actatropica.2014.09.004

13. Lamberton PH, Kabatereine NB, Oguttu DW, Fenwick A, Webster JP. Sensitivity and specificity of multiple Kato-Katz thick smears and a circulating cathodic antigen test for Schistosoma mansoni diagnosis pre- and post-repeatedpraziquantel treatment. PLoS Negl Trop Dis (2014) 8(9):e3139. doi:10.1371/ journal.pntd.0003139

14. Assefa LM, Crellen T, Kepha S, Kihara JH, Njenga SM, Pullan RL, et al. Diagnostic accuracy and cost-effectiveness of alternative methods for detection of soil-transmitted helminths in a post-treatment setting in western Kenya. PLoS Negl Trop Dis (2014) 8(5):e2843. doi:10.1371/journal.pntd.0002843

15. de Vlas SJ, Engels D, Rabello AL, Oostburg BF, Van Lieshout L, Polderman AM, et al. Validation of a chart to estimate true Schistosoma mansoni prevalences from simple egg counts. Parasitology (1997) 114(Pt 2):113-21. doi: $10.1017 /$ S0031182096008207

16. de Vlas SJ, Gryseels B, Van Oortmarssen GJ, Polderman AM, Habbema JD. A model for variations in single and repeated egg counts in Schistosoma mansoni infections. Parasitology (1992) 104(Pt 3):451-60. doi:10.1017/ S003118200006371X

17. Degarege A, Legesse M, Medhin G, Teklehaymanot T, Erko B. Day-to-day fluctuation of point-of-care circulating cathodic antigen test scores and faecal egg counts in children infected with Schistosoma mansoni in Ethiopia. BMC Infect Dis (2014) 14:210. doi:10.1186/1471-2334-14-210

18. Vinkeles Melchers NV, van Dam GJ, Shaproski D, Kahama AI, Brienen EA, Vennervald BJ, et al. Diagnostic performance of Schistosoma real-time PCR in urine samples from Kenyan children infected with Schistosoma haematobium: day-today variation and follow-up after praziquantel treatment. PLoS Negl Trop Dis (2014) 8(4):e2807. doi:10.1371/journal.pntd.0002807

19. Koukounari A, Donnelly CA, Moustaki I, Tukahebwa EM, Kabatereine NB, Wilson S, et al. A latent Markov modelling approach to the evaluation of circulating cathodic antigen strips for schistosomiasis diagnosis pre- and postpraziquantel treatment in Uganda. PLoS Comput Biol (2013) 9(12):e1003402. doi:10.1371/journal.pcbi.1003402

20. Sousa-Figueiredo JC, Betson M, Kabatereine NB, Stothard JR. The urine circulating cathodic antigen (CCA) dipstick: a valid substitute for microscopy for mapping and point-of-care diagnosis of intestinal schistosomiasis. PLoS Negl Trop Dis (2013) 7(1):e2008. doi:10.1371/journal.pntd.0002008
21. Colley DG, Binder S, Campbell C, King CH, Tchuem Tchuenté LA, N'Goran $\mathrm{EK}$, et al. A five-country evaluation of a point-of-care circulating cathodic antigen urine assay for the prevalence of Schistosoma mansoni. Am J Trop Med Hyg (2013) 88(3):426-32. doi:10.4269/ajtmh.12-0639

22. Coulibaly JT, Knopp S, N'Guessan NA, Silué KD, Fürst T, Lohourignon LK, et al. Accuracy of urine circulating cathodic antigen (CCA) test for Schistosoma mansoni diagnosis in different settings of Côte d'Ivoire. PLoS Negl Trop Dis (2011) 5(11):e1384. doi:10.1371/journal.pntd.0001384

23. Corstjens PL, De Dood CJ, Kornelis D, Fat EM, Wilson RA, Kariuki TM, et al. Tools for diagnosis, monitoring and screening of Schistosoma infections utilizing lateral-flow based assays and upconverting phosphor labels. Parasitology (2014) 141(14):1841-55. doi:10.1017/S0031182014000626

24. Zhang YY, Luo JP, Liu YM, Wang QZ, Chen JH, Xu MX, et al. Evaluation of KatoKatz examination method in three areas with low-level endemicity of schistosomiasis japonica in China: A Bayesian modeling approach. Acta Trop (2009) 112(1):16-22. doi:10.1016/j.actatropica.2009.05.020

25. Fleiss JL. Measuring nominal scale agreement among many raters. Psychol Bull (1971) 76(5):378-82. doi:10.1037/h0031619

26. Landis JR, Koch GG. The measurement of observer agreement for categorical data. Biometrics (1977) 33:159-74. doi:10.2307/2529310

27. Dendukuri N, Bélisle P, Joseph L. Bayesian sample size for diagnostic test studies in the absence of a gold standard: comparing identifiable with non-identifiable models. Stat Med (2010) 29(26):2688-97. doi:10.1002/sim.4037

28. Carneiro TR, Pinheiro MC, de Oliveira SM, Hanemann AL, Queiroz JA, Bezerra FS. Increased detection of schistosomiasis with Kato-Katz and SWAP-IgG-ELISA in a Northeastern Brazil low-intensity transmission area. Rev Soc Bras Med Trop (2012) 45(4):510-3. doi:10.1590/S0037-86822012000400019

29. Booth M, Vounatsou P, N'Goran EK, Tanner M, Utzinger J. The influence of sampling effort and the performance of the Kato-Katz technique in diagnosing Schistosoma mansoni and hookworm co-infections in rural $\mathrm{Co}^{\wedge}$ te d'Ivoire. Parasitology (2003) 127:525-31. doi:10.1017/S0031182003004128

30. Knopp S, Rinaldi L, Khamis IS, Stothard JR, Rollinson D, Maurelli MP, et al. A single FLOTAC is more sensitive than triplicate Kato-Katz for the diagnosis of low intensity soil-transmitted helminth infections. Trans $\mathrm{R}$ Soc Trop Med Hyg (2009) 103:347-54. doi:10.1016/j.trstmh.2008.11.013

31. da Frota SM, Carneiro TR, Queiroz JA, Alencar LM, Heukelbach J, Bezerra FS. Combination of Kato-Katz faecal examinations and ELISA to improve accuracy of diagnosis of intestinal schistosomiasis in a low-endemic setting in Brazil. Acta Trop (2011) 120(Suppl 1):S138-41. doi:10.1016/j.actatropica.2010. 05.007

32. Enk MJ, Lima AC, Drummond SC, Schall VT, Coelho PM. The effect of the number of stool samples on the observed prevalence and the infection intensity with Schistosoma mansoni among a population in an area of low transmission. Acta Trop (2008) 108(2-3):222-8. doi:10.1016/j.actatropica.2008. 09.016

33. Kongs A, Marks G, Verlé P, Van der Stuyft P. The unreliability of the KatoKatz technique limits its usefulness for evaluating S. mansoni infections. Trop Med Int Health (2001) 6(3):163-9. doi:10.1046/j.1365-3156.2001.00687.x

34. Gryseels B, Mbaye A, De Vlas SJ, Stelma FF, Guissé F, Van Lieshout L, et al. Are poor responses to praziquantel for the treatment of Schistosoma mansoni infections in Senegal due to resistance? An overview of the evidence. Trop Med Int Health (2001) 6(11):864-73. doi:10.1046/j.1365-3156.2001.00811.x

35. Kitange HM, Swai AB, McLarty DG, Alberti KG. Schistosomiasis prevalence after administration of praziquantel to school children in Melela village, Morogoro region, Tanzania. East Afr Med J (1993) 70(12):782-6.

36. Picquet M, Vercruysse J, Shaw DJ, Diop M, Ly A. Efficacy of praziquantel against Schistosoma mansoni in northern Senegal. Trans R Soc Trop Med Hyg (1998) 92(1):90-3. doi:10.1016/S0035-9203(98)90971-3

37. Ojurongbe O, Sina-Agbaje OR, Busari A, Okorie PN, Ojurongbe TA, Akindele AA. Efficacy of praziquantel in the treatment of Schistosoma haematobium infection among school-age children in rural communities of Abeokuta, Nigeria. Infect Dis Poverty (2014) 1(3):30. doi:10.1186/2049-9957-3-30

Conflict of Interest Statement: The authors declare that the research was conducted in the absence of any commercial or financial relationships that could be construed as a potential conflict of interest.

Received: 19 February 2015; accepted: 04 March 2015; published online: 19 March 2015. 
Citation: Mwinzi PNM, Kittur N, Ochola E, Cooper PJ, Campbell CH Jr., King $\mathrm{CH}$ and Colley DG (2015) Additional evaluation of the point-of-contact circulating cathodic antigen assay for Schistosoma mansoni infection. Front. Public Health 3:48. doi: $10.3389 / f p u b h .2015 .00048$

This article was submitted to Infectious Diseases, a section of the journal Frontiers in Public Health.
Copyright () 2015 Mwinzi, Kittur, Ochola, Cooper, Campbell, King and Colley. This is an open-access article distributed under the terms of the Creative Commons Attribution License (CC BY). The use, distribution or reproduction in other forums is permitted, provided the original author(s) or licensor are credited and that the original publication in this journal is cited, in accordance with accepted academic practice. No use, distribution or reproduction is permitted which does not comply with these terms. 